\title{
COVID-19 has intensified existing intergenerational and gender tensions in China's cities
}

Edition 8, 2021

Bin Wang \& Ilan Wiesel

DOI: 10.37839/MAR2652-550X8.1

On the $25^{\text {th }}$ of January 2020, China's National Health Commission declared the city of Wuhan the epicentre of the outbreak of COVID-19. Soon afterwards, most provinces in China introduced the highest category of restrictions, including strict limitations on people's movement in and out of communities and homes.

We examined the impact of these public health restrictions on the social relations of selected households in the city of Quanzhou, China. We found that COVID-19 and the resulting public health measures played a critical role in intensifying and surfacing existing tensions within households.

We justify this urban focus because most of the Chinese population resides in cities, and also because urban residents experienced some of the toughest restrictions during the period of late January to mid-March 2020.

This article reports on a study that involved a total of 20 interviews conducted in Quanzhou, a middle-sized city in the province of Fujian. These interviews were undertaken in June 2020, after the city achieved some success in controlling local outbreaks, and restrictions on Quanzhou residents were loosened. The primary focus for data collection and analysis was changes in people's everyday social relations during the period when they were confined to their homes under lockdown. Recent 
evidence shows that the pandemic has become an important driver of tensions within families. We explore how people's relationships changed in the private sphere during the outbreak.

All interviews were semi-structured, and interviewees were encouraged to share perspectives and stories from their lockdown experience as freely as possible within the broad parameters described above. Participants were recruited through convenience and snow-ball sampling. Since 'family issues' are commonly perceived as a private matter in traditional Chinese culture, social connections between the researcher and the interviewee played a significant role in facilitating the process of data collection. Furthermore, purposive sampling was also used to ensure the sample included participants of different ages, social backgrounds, occupations, gender, and sexuality.

\section{Conflicts: unexpected family time}

Like the rest of China, Quanzhou witnessed a major COVID-19 outbreak at the time of Spring Festival. Other than being the longest public holiday in China, it is also an important time for family reunion in traditional Chinese culture. Consequently, when the lockdown was announced, some participants were forced to stay with their families for a far longer time than they would have chosen otherwise:

'I work in Shanghai, so I came back home only for (Chinese) New Year holiday and the pandemic just happened... I did not expect to stay with them that long and I don't like it.' (Interviewee 6, 27 years, female)

This coincidental timing of a pandemic during the Spring Festival created conditions that proved conducive to changes in familial relations. In particular, all of the younger participants in this research reported experiencing conflict with their parents during the lockdown at a frequency and intensity that they considered to be higher than usual: 
'When the quarantine occurred, we were forced to stay inside together. It (conflict) was unavoidable. We [the interviewee and his parents] just had to talk to each other more often. They like to ignore or deny my opinions and that usually ended up as arguments. Even if I was in my room, they would just come in and start nagging, about my grade[s] in university or some other things they don't like about me...' (Interviewee 10, 22 years, male)

'I have been planning to go to Beijing for work after Chinese New Year and they [parents] hate that idea. We had discussions before the pandemic but did not come to any agreement. Then the lockdown occurred... they brought up the topic every time we sat together, and you can imagine what would happen.' (Interviewee 1, 24 years, female)

There was a generational difference in how interviewees spoke about such conflicts. Older interviewees showed some reluctance to acknowledge conflicts within their family. For example, Interviewee 12 (48 years, female) denied the occurrence of 'arguments' with her son during the lockdown and referred those as 'intense conversations'. Younger participants were more open to directly acknowledge conflicts with their parents. This reflects perhaps the traditional view that family affairs are a private matter, and that younger generations have turned away from this view.

A clear distinction was evident in how young participants resolved conflicts with their parents: those who were already in the labour market and capable of supporting themselves financially, tended to confront and argue against their parents when disagreements escalated. In contrast, participants who were still students and financially dependent on their parents preferred to avoid arguments or stay silent when disagreements occurred.

All participants, regardless of age, stated that the pandemic and the lockdown were the reason for tension in the family. Phrases like 'if not for the pandemic' and 'it was all because of the pandemic' were uttered many times in these interviews. 
Interviewee 10, for instance, stated that he would not have become involved in any arguments with his parents if 'it had been a normal Chinese New Year just like before'.

However, when younger interviewees reflected more deeply on their interactions with parents, often a different understanding would emerge:

'They [parents] argued with me over the same things... how messy my room is, why I eat out with my friends so often, why stay up so late playing games... it was just more frequent during the lockdown.' (Interviewee 2, 24 years, male)

'They [parents] love to know about my love life... they really want me to find someone and get married soon, but I don't. If I tell them my true feelings about this, they would keep nagging, at a certain point I just could not hold it and it turned into arguments.' (Interviewee 6, 27 years, female)

Both quotes suggest that despite an increase in the frequency and intensity of family conflicts during the lockdown, the issues underlying those arguments existed before the pandemic. Frequently mentioned contentious issues included academic performance, career planning, and marriage or relationship status. Younger men (Interviewees 2, 9, 10 and 19) reported getting involved in conflict with their parents on various issues across this spectrum. But for these younger male participants, disagreement over any specific issue was regarded as a reflection of a wider power imbalance in their relationship with their parents, and irreconcilable generational differences in values and lifestyles. In contrast, all younger female interviewees reported that most of their arguments with parents related to one specific issue: marriage.

Previous studies have demonstrated that although the social and cultural norms of marriage have undergone radical transformation in China over past decades, the centrality of marriage and family formation remains robust. However, for younger women in contemporary China, increased opportunities for higher education, and greater earning capacity and economic independence often clash with the persisting 
social expectation that they embrace domesticity and traditional notions of womanhood. Younger women's changing understanding of marriage, as an option rather than obligation, challenges traditional gender norms, leading to disagreements or even conflict with parents, in a societal transformation that began long before the COVID-19 pandemic.

Previous studies on social structure in contemporary China illuminate some additional underlying factors behind the exacerbated conflict observed during the COVID-19 lockdown. Scholars Xiaoying Qi and Yida Zhai suggest that although Chinese society has experienced radical and transformative socio-cultural change since the 1990s, parental authority is still a dominant component of contemporary family structure. The authority of the parent is exerted in almost all aspects of family relations, and the autonomy of young adults continues to be restricted by their parents' expectations and preferences.

This traditional norm is believed to be rooted in Confucianism, which portrays filial piety as an essential quality for all individuals. Filial piety refers to the virtue of respect and obedience towards one's parents, elders and ancestors. Within the family, the fundamental social unit in Confucianism, parent-child relations are the micro-representation of the wider superordinate-subordinate filial piety model. In recent decades, however, this code of conduct is gradually losing its grip among the younger generations. The rise of individualism, in which the individual is regarded as the basic unit of society, has challenged the foundations of parental domination. The two clashing ideologies of Confucianism and individualism have thus created a generational divide, and the potential for conflict between young adults and their parents about marriage:

'It is pointless to argue, because they (parents) just won't listen to me and they won't understand why young people nowadays don't think of marriage as something we have to do. Even if I explain, they still won't accept it.' (Interviewee 3, 26 years, female) 
'Of course I nag, because I care about my child! In my generation, we believe everyone needs to get married and raise a child...that is the right way to live, and as a parent I want my child to be on the right path.' (Interviewee 15, 55 years, female)

While COVID-19 lockdowns throughout Spring Festival in 2020 created conditions for direct conflict to emerge between parents and young adults, the underlying tension between parental authority and young adults' autonomy has far deeper roots in the culture and history of Chinese society.

\section{Spousal relations in crisis: hidden gender tensions made visible}

Interviewee 7 (26 years, female) provided critical insights into the pandemic lockdown and its influence on gender roles and relations in the private sphere. She was in a three-year relationship with her partner, and they had been living together in a rental apartment for almost two years, when the lockdown began. A series of arguments occurred between the interviewee and her partner during the lockdown, which made her initiate a break-up afterwards. The direct trigger for these arguments was the unequal allocation of housework: according to the interviewee, her partner regarded her as responsible for all housework during the lockdown. While she had undertaken housekeeping since they moved in together, what frustrated her most during lockdown was the realisation that her partner was fully capable and available to offer a hand, yet he would 'sit on the sofa playing games'. What she had once been willing to accept, had become unacceptable:

'Normally he did not have the time to help me, but he was in the house with me all the time during lockdown! Just imagine the feeling that you woke up in the morning and then cooking, washing dishes...non-stop for hours, and you tried to stretch your back because you are so tired, then seeing him lying on the sofa playing games...'

Although the interviewee made attempts to communicate her frustration and change 
her partner's behaviour, her efforts had failed. As a result, she terminated the relationship after the lockdown was lifted:

'I could see exactly what my life will be like for the next decades if I stay with him, and it would be even harder if we had a child. I will need to do all the housework and take care of the child... that's not acceptable.'

In this participant's narrative, the role of financial contributions to the household was highlighted several times. To her, the unequal allocation of housework would have been more acceptable if her partner were fully responsible for their earnings. This perspective is rooted in traditional Chinese gender norms, where men are responsible for issues 'outside' the home such as breadwinning, while women are assigned to domestic responsibilities 'inside' the home, such as housekeeping. However, Binli Chen suggests that this gendered model for spousal relations has lost some of its currency in contemporary China. Increased education and labour force participation, as well as growing awareness of gender equity have allowed and encouraged more young women to seek financial independence. Therefore, resistance to unequal gender norms in the domestic environment is made possible, and more young women expect housework to be assigned fairly between them and their male partners, just like financial earning. Although Interviewee 7's financial contribution to the household was smaller than her partner's, she expected him to reciprocate by taking on an appropriate portion of housework. Lockdown exposed this unevenness, leading to the termination of their relationship.

A generational difference was observed in the way other interviewees managed similar spousal conflicts. Interviewee 11 (49 years, female) and Interviewee 12 (48 years, female) also reported that they undertook all housework while also financially supporting the family. The former expressed negative feelings when talking about how her partner failed to assist with the growing amount of housework during lockdown. Nonetheless, both participants suggested that they have become accustomed to, and accepted, the role assigned to them. Therefore, they avoided direct confrontation with their male partners on this matter. Likewise, Interviewee 
20 's (60 years, male) perspective on the proper distribution of housework also reflected the older generation's orthodoxy on this topic:

'Men go out and earn money. Women stay in and take care of the family...of course, it will be their (women's) responsibility to do the housework. This is natural and spontaneous, and it should always be like that, even for young people like you.'

\section{How has COVID-19 impacted to social relations in China?}

Most participants considered the pandemic to be the source of conflicts experienced in their social relations in the private sphere. However, as we engaged more carefully with their stories, a more complex picture emerged: the roots of those conflicts which seemed to have been triggered by COVID-19, existed well before the crisis. Yet, COVID-19 did play a role in altering social relations, albeit in more complex and ambiguous ways, by intensifying and surfacing already-existing tensions.

The narratives of intergenerational domestic conflict illustrate how the pandemic and the lockdown brought existing tensions to a boiling point. The excessive amount of family time that resulted from the lockdown, has intensified existing unsettled tensions. Issues that could have been left unresolved under ordinary circumstances, such as clashing ideas about marriage, parental authority, and vastly different lifestyles across generations, erupted into open verbal conflict. In other times, young adults would have been able to avoid conflict by steering clear of argument with their parents, living a big part of their lives away from their parents' gaze. However, the lockdown dismantled this strategy, since many were forced to stay with their family in a compressed space over an extended period. In such circumstances, confrontation and intensified conflict were often unavoidable (at least for young adults who achieved some financial independence from their parents). Likewise, Interviewee 7's story on a spousal relationship breakdown provided insight into the 
'surfacing' impacts of COVID-19. Gender inequality had always been a part of her spousal relationship, yet surfaced in a more visible way during the lockdown. The visibility of gender inequality in her relationship drove her to take action for change.

\section{COVID-19 and onwards: gender transitions in China}

Are changing gender relations in China moving in progressive and regressive ways? And in which direction is COVID-19 tipping the balance? The country's declining status on the Global Gender Gap Index since 2010, is a cause for pessimism. Indeed, recent policies such as the three-child policy announced in May 2021, have sparked nation-wide debate and concerns about the negative impacts on gender equality, with women expected to carry most of the added care burden.

The perspectives of our interviewees, however, offer a more ambiguous perspective. While older female participants accepted a higher burden of care work, the story of Interviewee 7, who broke up with her partner because of the unfair distribution of domestic work, points to resistance among young women. This resistance has been triggered and intensified during COVID-19 lockdowns. Therefore, while the upcoming post-pandemic or pandemic-as-new-normal era is likely to become an important turning point for gender relations, whether Chinese society is set to return to more traditional gender norms, or to move forward towards greater gender equality, remains uncertain and open.

Authors: Bin Wang \& Ilan Wiesel.

Image: Rooftops in Quanzhou. Credit: Tommy Wong/Flickr 